\title{
Extracellular Stimulation Window Explained by a Geometry-Based Model of the Neuron-Electrode Contact
}

\author{
Jan Reinoud Buitenweg*, Wim L. C. Rutten, and Enrico Marani
}

\begin{abstract}
Extracellular stimulation of single cultured neurons which are completely sealing a microelectrode is usually performed using anodic or biphasic currents of at least $200 \mathrm{nA}$. However, recently obtained experimental data demonstrate the possibility to stimulate a neuron using cathodic current pulses with less amplitude. Also, a stimulation window is observed. These findings can be explained by a finite-element model which permits geometry-based electrical representation of the neuron-electrode interface and can be used to explore the required conditions for extracellular stimulation in detail. Modulation of the voltage sensitive channels in the sealing part of the membrane appears to be the key to successful cathodic stimulation. Furthermore, the upper limit of the stimulation window can be explained as a normal consequence of the neuronal membrane electrophysiology.
\end{abstract}

Index Terms-Action potentials, extracellular stimulation, finite-element modeling, multielectrode arrays, neuron-electrode contact, voltage-sensitive channel densities.

\section{INTRODUCTION}

$\mathbf{P}$ LANAR microelectrode arrays (MEAs) have become a common tool for establishing electrical contacts with cultured neurons. The use of MEAs as two-way neuroelectronic interfaces not only requires excellent recording properties but also reliable and selective stimulation of cultured neurons. Single neurons which are completely sealing a microelectrode would be highly suitable for these purposes [1].

Although successful extracellular stimulation by application of both current and voltage pulses is reported in literature, the applied stimulus amplitudes are spread over a wide range. Regehr et al. could stimulate cultured large neurons from an Helisoma (soma diameter $>60 \mu \mathrm{m}$ ) with a diving board electrode using current pulses of $200 \mathrm{nA}$ during $200 \mu$ s [1]

Manuscript received November 1, 2001; revised June 20, 2002. This work was part of the NESTING Project (Neuro-Electronic Systems toward Tissue Implantation by Neural Grafting) and supported by the BIOMED II EC Project, shared cost Contract BMH4-2723 and also by the OSF-pioneer fund of the University of Twente. Asterisk indicates corresponding author.

*J. R. Buitenweg is with the Institute for Biomedical Technology, Signals and Systems Group, Faculty of Electrical Engineering, University of Twente, P.O. Box 217, 7500 AE Enschede, The Netherlands (e-mail: J.R.Buitenweg@el.utwente.nl)

W. L. C. Rutten is with the Institute for Biomedical Technology, Signals and Systems Group, Faculty of Electrical Engineering, University of Twente, 7500 AE Enschede, The Netherlands.

E. Marani is with the Institute for Biomedical Technology, Signals and Systems Group, Faculty of Electrical Engineering, University of Twente 7500 AE Enschede, The Netherlands. He is also with the Neuroregulation Group, Department of Neurosurgery, Leiden University Medical Center, Leiden, The Netherlands.

Digital Object Identifier 10.1109/TBME.2002.804504

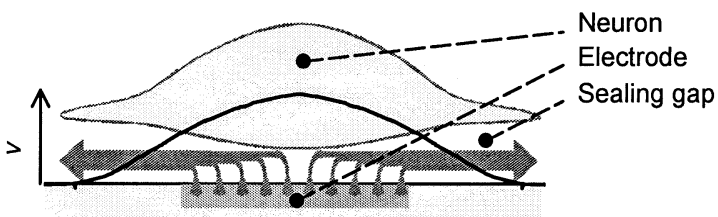

Fig. 1. Mediation of the neuron-electrode interface in extracellular stimulation. Due to current densities, arising from the electrode, a potential distribution exists in the sealing gap which modifies the membrane potential.

Breckenridge et al. also used large neurons from an invertebrate, but needed $500 \mathrm{nA}$ for successful stimulation with substrate embedded microelectrodes [2]. Much larger stimulus amplitudes, up to $15 \mu \mathrm{A}$, are reported for smaller neurons from vertebrates (diameters up to $15 \mu \mathrm{m}$ ) [3]-[6]. Stimulation with brief voltage pulses $(100 \mu \mathrm{s})$ required amplitudes of $0.6-2.5 \mathrm{~V}$, which could also result in current peaks of several microampères [7]-[10]. The shapes of these current and voltage pulses were anodic, resulting in positive stimulation currents, and biphasic. However, in previous work it was demonstrated that cathodic, i.e., negative, current pulses not only results in successful stimulation, but also in a decreased stimulation threshold of $20 \mathrm{nA}$ [11]. Furthermore, for eliciting action potentials an upper limit between 60 and $80 \mathrm{nA}$ was observed, indicating the existence of an amplitude window for successful stimulation. This stimulation window was not reported before, for cases of complete sealing.

The interpretation of stimulation results is hampered by the lack of detailed understanding of the electrical contact between the neuron and the electrode, as also stated by Jenkner et al. [12]. So far, it is generally recognized that when a neuron seals an electrode completely, an extracellular stimulation current will cause a potential distribution in the sealing gap between the neuronal membrane and the substrate (Fig. 1). Due to this potential distribution, the membrane potential will be modified locally resulting in depolarized and hyperpolarized regions [1], [6]. Initiation of an action potential by the opening of voltage sensitive sodium channels requires a region of the membrane which is depolarized with $15-30 \mathrm{mV}$. This region can be located in the sealing part of the membrane or in the upper part, depending on the direction of the stimulation current. The inward sodium current through the initially opened channels will depolarize the rest of the membrane, toward full development of the action potential [6]. However, the action potential will not evolve if the sodium current provides insufficient charge for this depolarization. The charge provided by the sodium current depends 


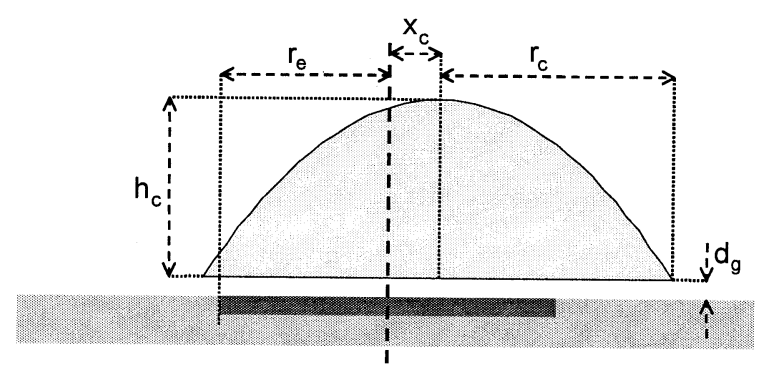

Fig. 2. Geometry of the modeled neuron-electrode interface.

on the number of opened sodium channels, the current contribution of each channel and the duration of these currents. This charge should exceed a critical quantity which depends on the area to be depolarized and the required depolarization which, in turn, depends on the initial hyperpolarization of this area and the threshold for channel opening. Hence, the conditions for extracellular stimulation of bioelectrical activity are a delicate mixture of influences from interface geometry and ion channel dynamics. Furthermore, it is not clear how these conditions can result in the stimulation window as observed in experimental data.

In order to gain insight into these conditions, the previously observed stimulation window is experimentally explored in more detail using several stimulus amplitudes and durations. The experimental data will be explained using a finite-element model (FEM) of the neuron-electrode interface, which includes the interface geometry, the conductivity of the sealing gap and the local membrane properties [13]. The model permits simulation of the local membrane potentials and current densities due to extracellular stimulation currents.

\section{METHODS}

\section{Experiments}

Dorsal root ganglia are dissected from neonatal (P3) rats, enzymatically dissociated (collagenase and trypsin) and plated on a MEA with 61 hexagonally ordered electrodes of $10 \mu \mathrm{m}$ in diameter (impedance 200-500 k $\Omega$ at $1 \mathrm{kHz}$ ). Both the MEA and the experimental setup are described in previous work [11]. From observation using phase contrast microscopy, electrodes are selected which are completely covered by a neuron. Sealing resistances are measured using impedance spectroscopy, also described in previous work [11], [14]. The intracellular potential of the neuron is measured using a whole cell current clamp configuration [15]. Pipette filling solution contains (in $\mathrm{mM}$ ): $140 \mathrm{KCl}, 10 \mathrm{NaCl}, 1 \mathrm{CaCl}_{2}, 2 \mathrm{MgCl}_{2}, 10$ EGTA, 10 HEPES 2 ATP-Mg, $\mathrm{pH}$ 7.2. The extracellular solution contains (in $\mathrm{mM}$ ): $140 \mathrm{NaCl}, 5 \mathrm{KCl}, 1 \mathrm{CaCl}_{2}, 6$ Glucose, $1 \mathrm{MgCl}_{2}, 10$ HEPES, $\mathrm{pH}$ 7.2. Extracellular current stimulation was applied by applying voltage pulses with a duration of $1 \mathrm{~ms}$ via a series resistance of $54.3 \mathrm{M} \Omega$ to the microelectrode. The intracellular and extracellular potentials are recorded and digitized during and after stimulation.

\section{Model}

In previous work, an FEM of the neuron-electrode interface is already presented in detail, assuming only passive membrane properties [13]. The mathematics of this model will be repeated briefly and extended by addition of voltage sensitive channels in the membrane.

The neuron is modeled as a circular soma of radius $r_{c}$, with a parabolic height $\left(h_{c}\right)$ profile (Fig. 2). The neuron is centered on top of the electrode with radius $r_{e}$ by setting the eccentricity to $x_{c}=0$. A sealing gap of thickness $d_{g}$ is modeled between the soma and the substrate or the electrode. The axon and dendrites of the neuron are not included in the model.

The medium surrounding the interface, including the sealing gap, is represented by a three-dimensional homogenous volume conductor. This volume is meshed with tetrahedral-shaped volume elements which permit numerical solutions of the Poisson equation

$$
\vec{\nabla} \cdot(\sigma \vec{\nabla} V)=0
$$

with $V$ the electrical potential and $\sigma=1.65 \mathrm{~S} / \mathrm{m}$ the conductivity of the medium. The nodes at the outer boundary of the meshed volume conductor are set to zero potential, representing a distant counter electrode.

The potentials at the membrane nodes are defined as transmembrane potentials, i.e., with respect to a single intracellular node with potential $v_{i}$, and represented by a vector $\vec{v}_{m}=\left[v_{m, 1} \cdots v_{m, N_{m}}\right]^{T}$ with $N_{m}$, the number of membrane nodes. Similarly, the nodes at the electrode surface are defined as trans-electrode-electrolyte potentials, i.e., with respect to a single node representing the electrode metal potential $v_{e}$, and represented by a vector $\vec{v}_{s}=\left[\begin{array}{lll}v_{s, 1} & \cdots & v_{s, N_{s}}\end{array}\right]^{T}$, with $N_{s}$, the number of nodes at the electrode surface. The local currents into the membrane and electrode nodes, represented by the vectors $\vec{i}_{m}$ and $\vec{i}_{s}$, respectively, can be expressed as the sum of contributions from the local potentials, i.e., $\vec{v}_{m}$ and $\vec{v}_{s}$, and the stimulation currents injected to the electrode node or the intracellular node, $i_{e}$ and $i_{i}$

$$
\left[\begin{array}{c}
\vec{i}_{m} \\
\vec{i}_{s}
\end{array}\right]=K_{v i} \cdot\left[\begin{array}{l}
\vec{v}_{m} \\
\vec{v}_{s}
\end{array}\right]+K_{i i} \cdot\left[\begin{array}{c}
i_{e} \\
i_{i}
\end{array}\right]
$$

with $K_{v i}$ and $K_{i i}$ derived from the meshed volume conductor. In this paper, extracellular stimulation is applied by injecting a current $i_{e}$ into the node representing the electrode metal. No current is injected into the intracellular node, so $i_{i}=0$. Furthermore, the electrode potential and intracellular potential are computed as

$$
\left[\begin{array}{l}
v_{i} \\
v_{e}
\end{array}\right]=K_{v v} \cdot\left[\begin{array}{l}
\vec{v}_{m} \\
\vec{v}_{s}
\end{array}\right]+K_{i v} \cdot\left[\begin{array}{l}
i_{i} \\
i_{e}
\end{array}\right]
$$

with $K_{v v}$ and $K_{i v}$ also derived from the meshed volume conductor.

Although it is known that the membrane of a DRG neuron can contain a large variety of different voltage sensitive ion channels [16]-[19], a relatively simple description of a chick dorsal root ganglion neuron is used as presented by Bove et al. [20]. Only one type of sodium channel and one type potassium channel is considered. Therefore, for each node $n=1 \cdots N_{m}$, the time 
derivative of the local membrane potential is computed from the local membrane current and the ion specific membrane current

$$
\begin{aligned}
\frac{d v_{m, n}}{d t}= & \frac{1}{A_{m, n} \cdot c_{m}}\left[i_{m, n}-g_{N a, n}\left(v_{m, n}-V_{N a}\right)\right. \\
& \left.-g_{K, n}\left(v_{m, n}-V_{K}\right)-\hat{g}_{C l, n}\left(v_{m, n}-V_{C l}\right)\right]
\end{aligned}
$$

with $A_{m, n}$ the membrane area represented by node $n$ and $c_{m}=$ $1 \mu \mathrm{F} / \mathrm{cm}^{2}$, the membrane capacity per unit area. In this equation, the local ion specific voltage sensitive conductances are computed as $g_{N a, n}=A_{m, n} \cdot \hat{g}_{N a, n} \cdot m_{n}^{3} \cdot h_{n}$ for sodium and $g_{K, n}=A_{m, n} \cdot \hat{g}_{K} \cdot n_{n}^{4}$ for potassium. Maximum conductivities $\hat{g}_{N a}=15 \mathrm{mS} / \mathrm{cm}^{2}, \hat{g}_{K}=3.2 \mathrm{mS} / \mathrm{cm}^{2}$ and $\hat{g}_{C l}=0.26$ $\mathrm{mS} / \mathrm{cm}^{2}$ are used and the reversal potentials are set to $V_{\mathrm{Na}}=$ $50 \mathrm{mV}, V_{K}=-82 \mathrm{mV}$ and $V_{C l}=-65 \mathrm{mV}$. The time derivatives of the local $\mathrm{Na}^{+}$inactivation and $\mathrm{K}^{+}$activation constants, $h_{n}$ and $n_{n}$, respectively, are computed as

$$
\begin{aligned}
\frac{d h_{n}}{d t} & =\frac{\lambda_{h}\left(h_{\infty}\left(v_{m, n}\right)-h_{n}\right)}{\tau_{h}\left(v_{m, n}\right)} \\
\frac{d n_{n}}{d t} & =\frac{\lambda_{n}\left(n_{\infty}\left(v_{m, n}\right)-n_{n}\right)}{\tau_{n}\left(v_{m, n}\right)}
\end{aligned}
$$

with $\lambda_{h}, \lambda_{n}, h_{\infty}(v), n_{\infty}(v), \tau_{h}(v)$, and $\tau_{n}(v)$ adopted from the above mentioned chick DRG model [20]. The local $\mathrm{Na}^{+}$ activation constant, $m_{n}$, is considered to be very fast and is, therefore, computed directly from $u_{m}$ as $m_{n}=m_{\infty}\left(v_{m, n}\right)$ according to the same model.

The constant-phase behavior of the electrode-electrolyte interface (as observed in experimental data [14], [21], [22]) is difficult to simulate in the time domain. Since platinum electrodes are used, a purely capacitive nature of the electrode is assumed for simplicity, and the time derivatives of the local potentials at the electrode surface are computed for each node, $n=1 \cdots N_{s}$

$$
\frac{d v_{s, n}}{d t}=\frac{i_{s, n}}{A_{s, n} \cdot c_{s}}
$$

with $A_{s, n}$ the electrode area represented by node $n$ and $c_{s}=$ $500 \mu \mathrm{F} / \mathrm{cm}^{2}$ the electrode capacity per unit area, based on an impedance of $400 \mathrm{k} \Omega$ at $1 \mathrm{kHz}$, of a platinized electrode of $10-\mu$ $\mathrm{m}$ diameter.

The total set of partial difference equations (PDEs) is solved numerically in MATLAB version 5.3 (The Mathworks Inc.), using a variable order solver, based on numerical differentiation formulas, (ODE15s) with a maximum time-step of $10 \mu \mathrm{s}$. The initial conditions at $t=0$ were taken $\vec{v}_{m}=-65 \mathrm{mV}$ and $\vec{v}_{s}=0$. After solving the set of PDEs, all ion specific and capacitive membrane currents can be extracted from the computed membrane potentials and channel activation constants.

\section{Simulations}

In Table I, the parameters of all modeled geometries are summarized. Also, the total membrane area and the passive contact parameters as obtained in previous work are listed [13].

Simulation of extracellular stimulation is performed by application of rectangular current pulses through the extracellular electrode node. The amplitude and the duration of the stimulus pulse are varied.
TABLE I

List OF GeOMETRIES USED FOR SimUlations, TOGETHER With THE CORRESPONDING SEALING RESISTANCES AS COMPUTED in Section IV, SeE Also Fig. 2

\begin{tabular}{cccccc}
\hline $\begin{array}{c}\text { Geometry } \\
\text { No. }\end{array}$ & $\begin{array}{c}\mathrm{x}_{\mathrm{c}} \\
{[\mu \mathrm{m}]}\end{array}$ & $\begin{array}{c}\mathrm{r}_{\mathrm{c}} \\
{[\mu \mathrm{m}]}\end{array}$ & $\begin{array}{c}\mathrm{r}_{\mathrm{e}} \\
{[\mu \mathrm{m}]}\end{array}$ & $\begin{array}{c}\mathrm{d}_{\mathrm{g}} \\
{[\mathrm{nm}]}\end{array}$ & $\begin{array}{c}\mathrm{R}_{\mathrm{j}} \\
{[\mathrm{M} \Omega]}\end{array}$ \\
\hline 1 & 0 & 7 & 5 & 10 & 5.60 \\
2 & 0 & 7 & 5 & 50 & 1.14 \\
3 & 0 & 7 & 5 & 100 & 0.58 \\
4 & 0 & 10 & 5 & 50 & 1.81 \\
5 & 0 & 15 & 3 & 50 & 3.50 \\
6 & 0 & 15 & 5 & 50 & 2.57 \\
7 & 0 & 15 & 10 & 50 & 1.25 \\
8 & 0 & 20 & 5 & 50 & 3.12 \\
9 & 12 & 20 & 5 & 50 & 2.29 \\
10 & 18 & 20 & 5 & 50 & 0.64 \\
\hline
\end{tabular}

\section{RESULTS}

\section{Experimental}

A cultured neuron which is sealing a microelectrode is selected for extracellular stimulation experiments. After establishing a whole cell current-clamp configuration with a glass micropipette, a resting membrane potential of $-68 \mathrm{mV}$ is measured. The sealing resistance of $R_{j}=1.41 \mathrm{M} \Omega$ is measured using impedance spectroscopy. Cathodic extracellular stimulus pulses of $1 \mathrm{~ms}$ are applied (Fig. 3, lower diagrams) and an amplitude of $-20 \mathrm{nA}$ results in a 3-mV hyperpolarization of the intracellular potential (Fig. 3, upper diagrams). When the stimulus amplitude is increased to $-40 \mathrm{nA}$, the response of the intracellular potential starts with a short period of hyperpolarization followed by a strong depolarization. The rate of depolarization increases when the intracellular potential reaches a value of about $-40 \mathrm{mV}$. Then an action potential develops. A similar response is measured with -60-nA stimulation. However, no action potential results from a stimulus of $-80 \mathrm{nA}$. Instead the rate of depolarization decreases after $1 \mathrm{~ms}$ and the intracellular potential never reaches a value of $-40 \mathrm{mV}$.

The observed upper and lower limits for successful stimulation depend on the duration of the stimulus pulse (Fig. 4). With an amplitude of $-40 \mathrm{nA}$, no action potentials could be obtained using pulses shorter than $0.8 \mathrm{~ms}$, which indicates that the lower stimulation limit increases with decreasing pulse duration. Similarly, the upper stimulation limit also depends on the pulse duration.

\section{Simulated Stimulus Window}

Stimulation windows, as observed in the experimental results can also be produced by the FEM (Fig. 5, lower diagrams). Due to the applied stimulation currents through the sealing gap, the potential in the sealing gap becomes negative with respect to the potential in the rest of the culture medium. As a consequence, the lower membrane potential is depolarized, while the upper membrane (determining the measured intracellular potential) is slightly hyperpolarized (top row). In the simulations, a stimulus current of $-10 \mathrm{nA}$ results in a subthreshold depolarization of the lower membrane, i.e., no channels are activated and the intracellular recording only contains a passive response to the stimulus pulse. Due to a stimulation current of $-20 \mathrm{nA}$ the depolarization becomes suprathreshold above the electrode. The 

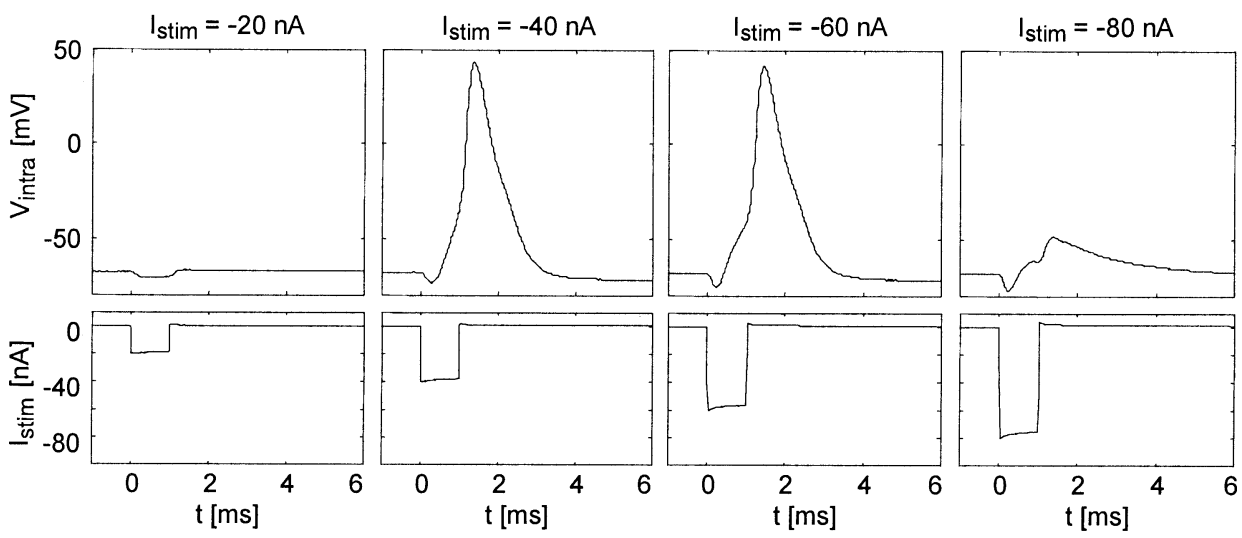

Fig. 3. Measured intracellular response to extracellular current stimulation (upper diagrams). A rectangular current pulse of $1 \mathrm{~ms}$ is applied at $t=0$ (lower diagrams). The stimulus amplitude is varied from -20 to $-80 \mathrm{nA}$ in four steps.

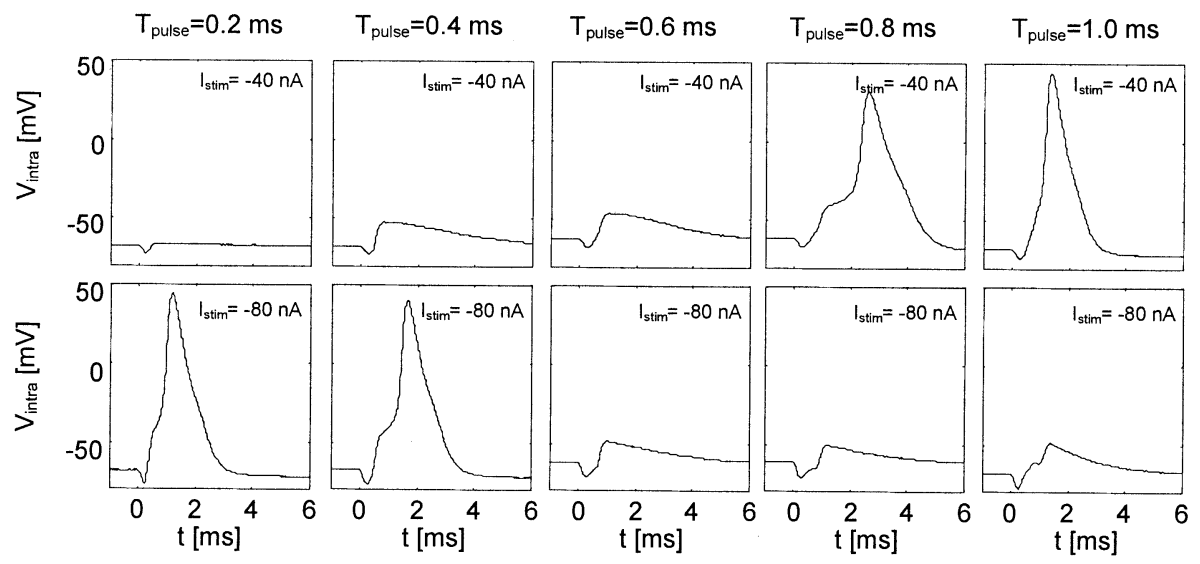

Fig. 4. Measured intracellular response to extracellular current stimulation. Stimulus currents of $-40 \mathrm{nA}$ (top row) and $-80 \mathrm{nA}$ (bottom row) are applied during 0.2 to $1.0 \mathrm{~ms}$.

opening of voltage-dependent sodium channels in this part of the membrane results in a local inward membrane current density (middle row). This current, in turn, depolarizes the upper membrane which can be observed from the intracellular potential. However, the current amplitude and duration are not sufficient for generation of an action potential. When the stimulus amplitude is increased to $-30 \mathrm{nA}$, the area of suprathreshold depolarization increases resulting in an increased inward sodium current. Due to this current, the intracellular potential can be depolarized sufficiently, and an action potential is generated. The width of the simulated action potential is much larger than the width of the experimentally obtained action potentials. This is due to the implemented channel dynamics, which differ from the experimental situation (slower $\mathrm{K}^{+}$delayed rectifier), as mentioned in the previous section. In a simulation with a stimulation amplitude of $-60 \mathrm{nA}$, the lower membrane above the electrode is depolarized to a value of $80 \mathrm{mV}$. Since this value exceeds the equilibrium potential for sodium $\left(V_{\mathrm{Na}}=50 \mathrm{mV}\right)$, opening of the voltage sensitive sodium channels will result in an outward instead of an inward current density! In the sealing gap around the electrode, the lower membrane potential radially decreases toward the potential of the upper membrane. An inward current density arises in a ring shaped region around the electrode where the membrane potential is below the sodium equilibrium potential but still above the threshold for opening of channels. Hence, the total inward membrane current is impaired by the outward current in the part of the membrane above the electrode. During the stimulation pulse, the upper membrane can no longer be depolarized far enough for initiation of an action potential.

\section{Pulse Duration}

The simulations with -20 and $-60 \mathrm{nA}$ stimuli in Fig. 5 suggest that a longer pulse duration would result in generation of an action potential. Therefore, the effect of pulse duration is studied in more detail for these stimulus amplitudes (Fig. 6). In case of a -20-nA stimulation pulse, longer pulses indeed result in a continued depolarization of the intracellular potential. An action potential is generated due to pulses of $2 \mathrm{~ms}$ and longer. However, this is not the case for stimulus pulse of $-60 \mathrm{nA}$. The initial depolarization is not continued during the pulse, but stops after $1.5 \mathrm{~ms}$, indicating a change in the inward lower membrane current during the stimulation pulse. After $1.5 \mathrm{~ms}$ it even turns into an outward hyperpolarizing current. The causes for this current inversion are explained in Fig. 7. The $\mathrm{Na}^{+}, \mathrm{K}^{+}$and total current densities at three time instants during the stimulus pulse of $2.5 \mathrm{~ms}$ and $-60 \mathrm{nA}$ are depicted. An outward $\mathrm{Na}^{+}$current density exists in the region above the electrode, while an inward current density exists in a ring shaped region around the electrode. The magnitude of the current density (inward and outward) decreases during the stimulus pulse, due to the inactiva- 


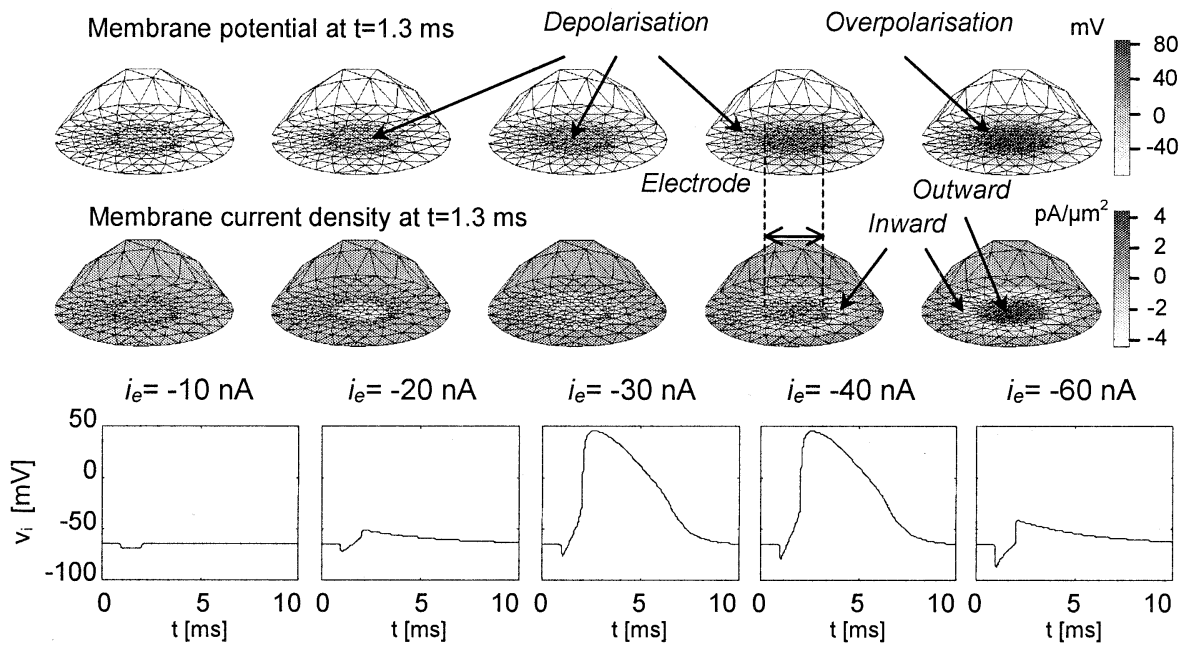

Fig. 5. Simulation of extracellular stimulation with several stimulus amplitudes (geometry number 6, see Table I). The intracellular responses to rectangular current pulses of $1 \mathrm{~ms}$ starting at $t=1 \mathrm{~ms}$, are plotted in the lower diagrams for stimulus amplitudes of $-10,-20,-30,-40$, and $-60 \mathrm{nA}$, respectively. For each stimulus amplitude, the distribution of local membrane potentials (top row) and the local membrane current densities (second row, a negative value denotes an inward current density) are depicted at $t=1.3 \mathrm{~ms}$.

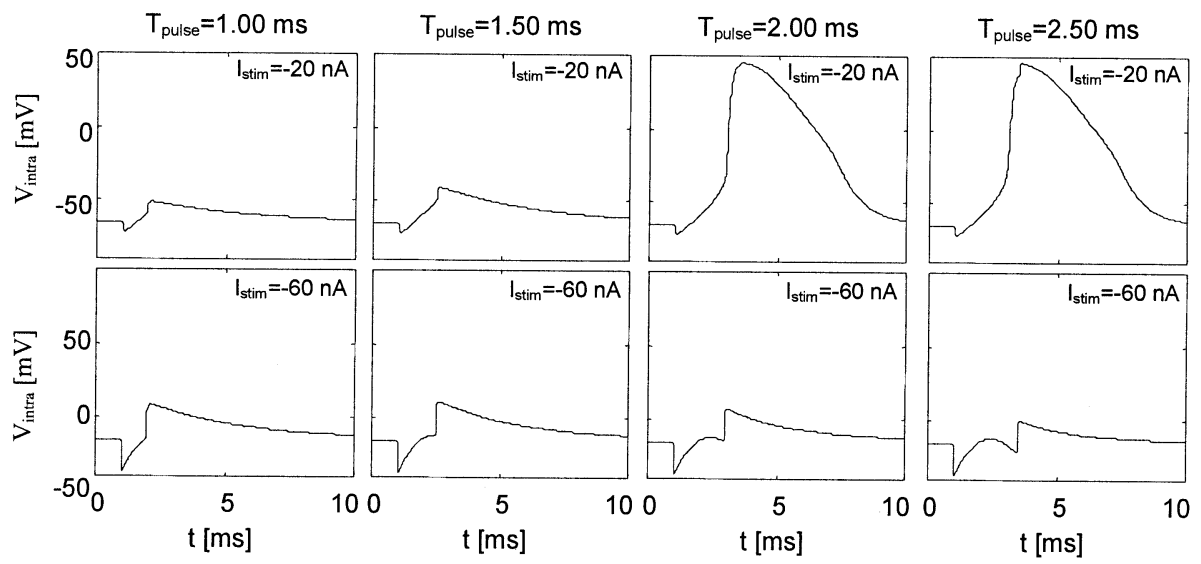

Fig. 6. Simulated intracellular responses for the same geometry as used in Fig. 5 to extracellular stimulation pulses of $-20 \mathrm{nA}$ (top row) and $-60 \mathrm{nA}$ (bottom row). The pulse width is varied from 1 to $2.5 \mathrm{~ms}$ in four steps.

tion of $\mathrm{Na}^{+}$channels. However, due to the opening of $\mathrm{K}^{+}$channels, an outward $\mathrm{K}^{+}$current density develops at the end of the stimulus pulse. As a consequence, the outward total membrane current density becomes more and more dominant over the inward current density which turns the depolarizing current in to a hyperpolarizing current through the membrane.

\section{Geometry}

The region of depolarization depends on the geometry of the neuron-electrode interface. Therefore, the influence of geometry on the upper and lower stimulation limit is studied using the geometries of Table I, for a pulse duration of $1 \mathrm{~ms}$. The success of stimulation is tested by applying stimulus amplitudes in steps of $-10 \mathrm{nA}$ For each geometry, Fig. 8(a) presents the amplitudes which resulted in the generation of an action potential. Variation of the sealing gap width from $d_{g}=10$ to $100 \mathrm{~nm}$ (geometries 1,2 and 3) has a large linear effect on both the upper and the lower stimulation limit. All other geometry parameters mainly affect the stimulation upper limit, leaving the lower limit between -30 and $-40 \mathrm{nA}$. An increase of the neuronal radius from $r_{c}=10$ to $20 \mu \mathrm{m}$ decreases the upper limit from -90 to
$-40 \mathrm{nA}$ (geometries 2, 4, 6, and 8). If the electrode radius is increased from 3 to $10 \mu \mathrm{m}$, the upper limit is increased from -40 to $-110 \mathrm{nA}$ (geometries 5,6 , and 7). No action potentials could be elicited when a large cell of radius $r_{c}=20 \mu \mathrm{m}$ was shifted away from the center of the electrode by increasing the eccentricity to $x_{c}=12 \mu \mathrm{m}$ (still complete sealing) and $x_{c}=18 \mu \mathrm{m}$ (defect sealing, geometries numbers 9 and 10, respectively).

For experimental purposes, it might be important to be able to make a prediction about the upper and the lower stimulation limit. Since the potential distribution in the sealing gap is proportional to the sealing resistance, the results from Fig. 8(a) are also used for exploring the relationship between stimulus limits and sealing resistance [Fig. 8(b)]. When the sealing resistance is higher than $1 \mathrm{M} \Omega$, the lower stimulation limit is between -20 and $-40 \mathrm{nA}$. The stimulation limits decreases with the sealing resistance in an inverse proportional manner as can be seen from a preliminary fit of a function $I_{\text {limit }}=K / R_{j}$ on these results. Assuming that the limits are in between two applied stimulus amplitudes (one successful, one not successful), the lower stimulation limit can be estimated using a value of $K=53.7 \mathrm{mV}$ and the upper limit using $K=136 \mathrm{mV}$. 

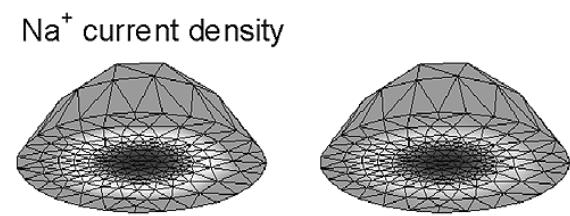

$\mathrm{K}^{+}$current density
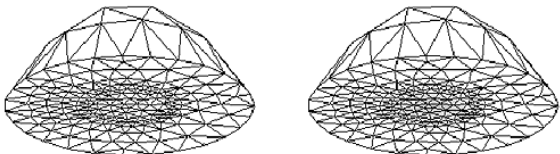

Total membrane current density

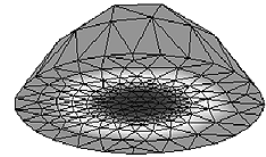

$t=1.5 \mathrm{~ms}$

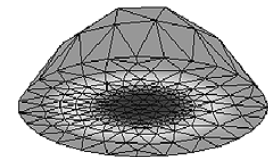

$t=2.0 \mathrm{~ms}$

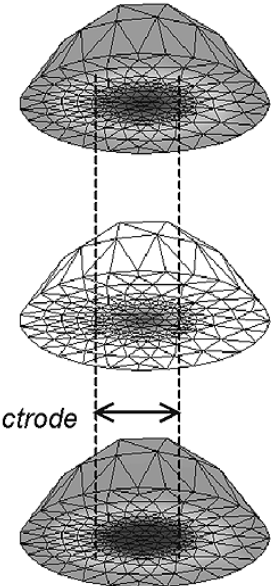

$t=2.5 \mathrm{~ms}$
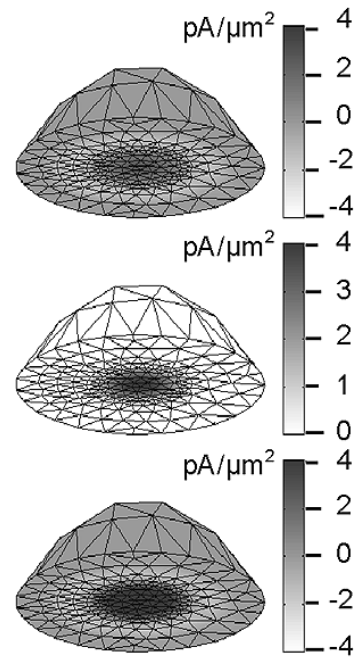

$t=3.0 \mathrm{~ms}$

Fig. 7. Local membrane current densities at several time instances $(t=1.5,2.0,2.5$, and $3.0 \mathrm{~ms})$ during a $2.5 \mathrm{~ms}$ stimulus pulse of $-60 \mathrm{nA}$ (e.g., Fig. 6 lower right diagram). First row: $\mathrm{Na}^{+}$current density distribution. Second row: $\mathrm{K}^{+}$current density distribution. Third row: Total membrane current density distribution.

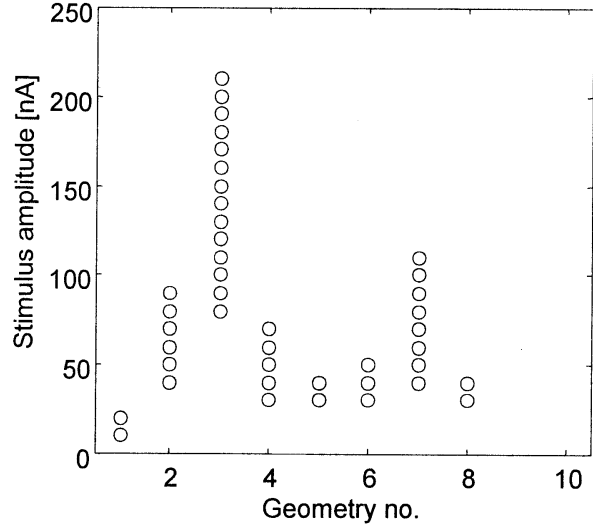

(a)

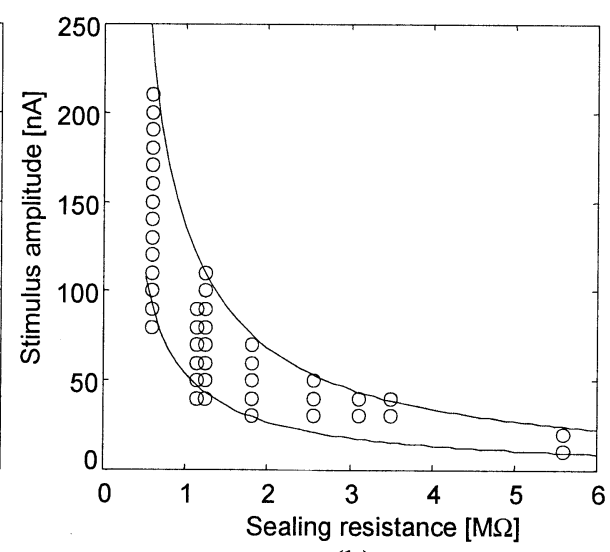

(b)

Fig. 8. (a) Influence of geometry on the stimulation window. The geometry numbers refer to the geometries listed in Table I and the stimulation results are obtained with a pulse duration of $1 \mathrm{~ms}$. Stimuli are applied in steps of $10 \mathrm{nA}$ and successful stimulations are plotted as a circle. (b) Relation between stimulation window and sealing resistance. The sealing resistances are extracted from Table I. A relationship $K / R_{j}$ between the sealing resistance and the upper $(K=136 \mathrm{mV})$ and lower $(K=53.7 \mathrm{mV})$ stimulation limit is plotted.

\section{DISCUSSION}

\section{Extracellular Stimulation Explored by FEM}

Finite element modeling is used to gain insight in the presented experimental results on extracellular stimulation. The model includes all coupling mechanisms involved in the neuron-electrode contact, which are: 1) volume conduction through the extracellular space, including the sealing gap; 2) electrical interaction of this volume conductor with the electrode-electrolyte interface; and 3) with the active neuronal membrane.

Although the used model parameters have close relevance to the physiological reality, the geometry of the experimental neuron-electrode interface is not exactly represented in the model. Furthermore, the modeled neuron is assumed to have only one type of sodium channel and one type of potassium channel. These assumptions are made only for instructive reasons, i.e., to gain insight in the general mechanisms of extracellular stimulation, not in a particular experimental situation. The model is not limited to these simplifications, but also permits representation of more realistic geometries, e.g., reconstructed from experimental data, and various types of channels in the neuronal membrane (which is beyond the scope of this paper).

As a result of this simplified use of the model, the duration of the simulated intracellular action potential is longer than the measured action potentials. Furthermore, the simulated stimulation limits differ from the experimental data. Except for these differences, the shapes and amplitudes of the simulated intracellular and extracellular potentials show similarity with the experimental data. This indicates that the simulation results demonstrate general mechanisms involved in cathodic stimulation, i.e., the effect of overpolarization, the combined action of multiple channels, and the influence of geometry.

The assumed capacitive behavior of the electrode-electrolyte interface does not totally agree with the experimentally observed impedances reported in literature [14], [21], [22]. This modeling assumption could weaken the model predictions 
if the electrode properties would have a large effect on the potential distribution in the sealing gap, which depends on the radial current density in the sealing gap and, hence, on the current density distribution arising from the electrode surface (Fig. 1). Fortunately, variation of the electrode capacity to reflect impedances of $200, \ldots, 800 \mathrm{k} \Omega$ at $1 \mathrm{kHz}$ did not result in large variations in the potential distribution (computed for geometry number 2 , results not shown). A slightly mismatched electrode impedance is, therefore, not likely to alter the effect of extracellular current stimulation as much as the mismatches in geometry and channel dynamics would do. Hence, as long as the modeled electrode behavior is in the range of the experimental data, improved prediction of the behavior of a particular neuron-electrode interface would primarily require proper reconstruction of the experimental interface geometry and proper representation of the neuronal channel dynamics.

\section{Modulation of Channels in the Lower Membrane}

The experimental results clearly demonstrate the possibility of successful cathodic stimulation and confirm the existence of the stimulation window as presented in previous work [11]. The observed response of the intracellular potential to a stimulus pulse of $-20 \mathrm{nA}$ indicates a hyperpolarization of the upper membrane, which is consistent with the direction of the stimulation current (Fig. 3). The depolarization of the intracellular potential, after initial hyperpolarization due to a stimulus amplitude of $-40 \mathrm{nA}$, must be caused by an active inward membrane current. Since the upper membrane is hyperpolarized and the lower membrane is depolarized, this current must be generated by opening of $\mathrm{Na}^{+}$channels in the lower membrane. This conclusion is confirmed by the simulations presented in Fig. 5. Although the geometry and the channel dynamics do not correspond to the experimental situation, it is clearly demonstrated how inward membrane current densities are generated in the depolarized area of the lower membrane resulting in a depolarization of the intracellular potential. The rate of depolarization depends on the number of open $\mathrm{Na}^{+}$channels and increases when the upper membrane is depolarized far enough for opening of channels.

Although the presence of voltage sensitive channels in the lower membrane has never been denied in literature, their possible mediating role for cathodic stimulation was not recognized before. The experimental and simulation results presented in this paper strongly indicate that proper modulation of lower membrane channels is the key to successful cathodic stimulation.

\section{Lower Stimulation Limit}

The observed lower amplitude limits are almost a magnitude lower than the smallest required amplitudes reported in literature for successful anodic stimulation [1], [2]. This should not surprise, since it is known that the lower membrane potential is influenced more strongly by stimulus current than the upper membrane potential [1], [12], [13], [23]. Unfortunately, this effect was interpreted by most investigators as a limiting factor for anodic stimulation currents due to danger of electrical breakthrough of the lower membrane.
As stated in Section I, for successful cathodic stimulation the total charge flowing through the channels in the lower membrane must be sufficient to depolarize the upper membrane to the threshold for opening of $\mathrm{Na}^{+}$channels. This charge depends on the amplitude and duration of the total current through the lower membrane which consists of the contributions from all individual channels. The simulation results demonstrate that the number of activated channels is modulated by the amplitude of the applied stimulus (Fig. 5). Hence, the stimulus amplitude modulates the total inward current through the lower membrane, which explains the different initial rates of depolarization (Fig. $5, i_{e}=-10,-20$, and $-30 \mathrm{nA}$ ).

If the initial rate of depolarization, i.e., the lower membrane current, can be maintained long enough, the intracellular potential will reach the threshold for opening of $\mathrm{Na}^{+}$channels in the upper membrane resulting in full development of an action potential. However, both the experimental and simulation results show that the depolarization stops if this threshold is not reached before termination of the stimulus current (Fig. 4, upper row and Fig. 6, upper row). This indicates that the activation of lower membrane channels is discontinued, as the potential distribution in the sealing gap no longer provides suprathreshold depolarization of the lower membrane. Theoretically, the inward currents generated by opened $\mathrm{Na}^{+}$channels themselves could induce a potential distribution sufficient for sustained opening of channels after termination of the stimulus. However, the requirements for this "self-gating" effect, as proposed by Fromherz, [24], are not met in these experiments and simulations. Therefore, the lower limit for successful stimulation depends on the duration of the stimulus pulse. Although FEM permits detailed analysis of the requirements for "self-gating" and the consequences for stimulation and recording, further considerations are beyond the scope of this paper.

\section{Upper Stimulation Limit}

The existence of a stimulation window might be somewhat counterintuitive and raise doubts about the validity of the experimental results. However, the simulation results demonstrate that, besides the lower stimulation limit, also the upper stimulation limit can be explained as a normal consequence of the membrane electrophysiology, as described in (4) and (5).

In the first place, the current contribution of opened $\mathrm{Na}^{+}$ channels to the total lower membrane current does not solely depend on the state of the channel, i.e., opened or closed, but also on the local membrane potential. Increase of the stimulus amplitude after opening of the channels will further depolarize the membrane, which decreases and even inverts the local inward $\mathrm{Na}^{+}$current densities, resulting in a strongly impaired total lower membrane current. As a consequence, the depolarization of the intracellular potential will no longer reach the threshold for full development of an action potential (Fig. 5, $i_{e}=-60 \mathrm{nA}$ ). Since further increase of the stimulus amplitude will enhance this effect, "overpolarization" of $\mathrm{Na}^{+}$channels in the lower membrane is a determining factor for the upper stimulation limit.

Another factor is the competition between currents generated by $\mathrm{Na}^{+}$and $\mathrm{K}^{+}$channels in the lower membrane. Increased de- 
polarization of the lower membrane will speed up the opening of $\mathrm{K}^{+}$channels, which results in an outward current density (Fig. 7). Together with the inactivation of $\mathrm{Na}^{+}$channels, the total lower membrane current will change from an inward (depolarizing) to an outward (hyperpolarizing) current. This explains the simulated decreasing depolarization rate during the stimulus pulse (Fig. 6), which is also observed in experimental results (Fig. 3, $I_{\text {stim }}=-80 \mathrm{nA}$ ).

\section{Significance for Future Experiments}

As mentioned in Section I, most investigators used anodic or biphasic stimulation instead of cathodic stimulation. Due to anodic stimuli, the upper membrane will be depolarized and an action potential will be initiated without the mediating role of channels in the lower membrane. As the intracellular potential is determined by the upper membrane potential, the stimulation threshold for anodic stimulation can be estimated from the initial hyperpolarization due to cathodic pulses. According to Fig. 3, a stimulus amplitude of $-20 \mathrm{nA}$ results in a hyperpolarization of $3 \mathrm{mV}$, which corresponds to $0.15 \mathrm{mV} / \mathrm{nA}$. Depolarization of the upper membrane from the resting membrane potential of $-68 \mathrm{mV}$ to the threshold for opening of $\mathrm{Na}^{+}$channels, which is about $-40 \mathrm{mV}$, would, therefore, require an anodic stimulus of about $187 \mathrm{nA}$. This is in the range of the amplitudes reported in literature [1], [2]. Due to this stimulus amplitude, a potential drop of about $264 \mathrm{mV}$ will exist over the sealing resistance, indicating a lower membrane hyperpolarization to at least $304 \mathrm{mV}$. Amplitudes exceeding $600 \mathrm{nA}$ will cause lower membrane potentials larger than $1 \mathrm{~V}$, which is the threshold for electrical membrane breakthrough. Hence, in case of complete sealing, cathodic stimulation is desirable above anodic stimulation.

The presence of a stimulation window increases the need for proper selection of stimulation parameters for reliable stimulation. Besides the risk of not reaching the lower limit, the possibility of exceeding the upper limit exists. Measurement of the sealing resistance, as can be performed using impedance spectroscopy, can provide useful information about the stimulus window, as appears from Fig. 8. Since sealing resistance can also be due to electrode coverage by nonneuronal cells, the pulse-response method for detection of active lower membrane currents [11], is a valuable addition for finding suitable stimulation parameters.

As the potential distribution in the sealing gap, which mediates in the modulation of the ionic currents through the lower membrane, depends on the geometry of the neuron-electrode interface, improvement of experimental cathodic stimulation results should focus on the "design" of this interface. Fig. 8 indicates that major progress can be obtained by decreasing the thickness of the sealing gap, e.g., by selection of proper adhesion promotors on the surface of the MEA or in the culture medium (geometries nos. 1,2, and 3). Other improvements may concern the size of the electrodes with respect to the size of the type of neurons investigated. Since the lower stimulation limit is only slightly affected by the size of the electrode, this size can be based on the desired width of the stimulation window, i.e., matched to the size of the cell (large window, geometries numbers 2 and 7) or taken as small as possible (small window, geometries numbers 5 and 8 ).

Although promising for reliable neuron-electrode interfaces, the neuron-electrode interfaces described in this paper are of very unique nature. Application of the results and insights presented in this paper toward more general neuron-electrode interfaces requires extrapolation to cases of 1) defect sealing and 2) neurons with axons and dendrites. Charged balance pulses are not required as long as the electrode-electrolyte potential does not induce chemical reactions $(<200 \mathrm{mV})$. However, in cases of defect sealing, much larger currents will be required for stimulation and chemical reactions will be induced more easily. Charge balancing will then be inevitable. Fortunately, the model also permits representation of geometries of defect sealing and simulation of any stimulation pulse shape, including bipolar shapes so the consequences of these changes for successful stimulation can be studied easily.

\section{ACKNOWLEDGMENT}

The authors would like to thank K. Peter and M. Deenen for their excellent support of the culturing activities in their lab and M. Goedbloed for the fabrication of the MEAs used in the experiments.

\section{REFERENCES}

[1] W. G. Regehr, J. Pine, and D. B. Rutledge, "A long-term in vitro siliconbased microelectrode-neuron connection," IEEE Trans. Biomed. Eng., vol. BME-35, pp. 1023-1032, 1988.

[2] L. J. Breckenridge, R. J. Wilson, P. Connolly, A. S. Curtis, J. A. Dow, S. E. Blackshaw, and C. D. Wilkinson, "Advantages of using microfabricated extracellular electrodes for in vitro neuronal recording," J. Neurosci. Res., vol. 42, pp. 266-276, 1995.

[3] Y. Jimbo and A. Kawana, "Electrical stimulation and recording from cultured neurons using a planar electrode array," Bioelectrochem. Bioenerg., vol. 29, pp. 193-204, 1992.

[4] Y. Jimbo, H. P. Robinson, and A. Kawana, "Simultaneous measurement of intracellular calcium and electrical activity from patterned neural networks in culture," IEEE Trans. Biomed. Eng., vol. 40, pp. 804-810, Aug. 1993.

[5] M. P. Maher, H. Dvorak-Carbone, J. Pine, J. A. Wright, and Y. C. Tai, "Microstructures for studies of cultured neural networks," Med. Biol. Eng. Comput., vol. 37, pp. 110-118, 1999.

[6] M. P. Maher, J. Pine, J. Wright, and Y. C. Tai, "The neurochip: A new multielectrode device for stimulating and recording from cultured neurons," J. Neurosc. Meth., vol. 87, pp. 45-56, 1999.

[7] Y. Jimbo, T. Tateno, and H. P. Robinson, "Simultaneous induction of pathway-specific potentiation and depression in networks of cortical neurons," Biophys. J., vol. 76, pp. 670-678, 1999.

[8] Y. Jimbo, A. Kawana, P. Parodi, and V. Torre, "The dynamics of a neuronal culture of dissociated cortical neurons of neonatal rats," Biol. $C y$ bern., vol. 83, pp. 1-20, 2000.

[9] T. Tateno and Y. Jimbo, "Activity-dependent enhancement in the reliability of correlated spike timings in cultured cortical neurons," Biol. Cybern., vol. 80, pp. 45-55, 1999.

[10] Y. Jimbo, H. P. Robinson, and A. Kawana, "Strengthening of synchronized activity by tetanic stimulation in cortical cultures: Application of planar electrode arrays," IEEE Trans. Biomed. Eng., vol. 45, pp. 1297-1304, Nov. 1998.

[11] J. R. Buitenweg, W. L. C. Rutten, and E. Marani, "Extracellular detection of active membrane currents in cultured neuron-electrode contacts," $J$. Neurosc. Meth., vol. 115, no. 2, pp. 211-212, 2002.

[12] M. Jenkner, B. Mueller, and P. Fromherz, "Interfacing a silicon chip to pairs of snail neurons connected by electrical synapses," Biol. Cybern., vol. 84, pp. 239-249, 2001.

[13] J. R. Buitenweg, W. L. C. Rutten, and E. Marani, "Geometry-based finite element modeling of the electrical contact between a cultured neuron and a microelectrode," IEEE Trans. Biomed. Eng., vol. 50, 2003, submitted for publication. 
[14] J. R. Buitenweg, W. L. Rutten, W. P. Willems, and J. W. van Nieuwkasteele, "Measurement of sealing resistance of cell-electrode interfaces in neuronal cultures using impedance spectroscopy," Med. Biol. Eng. Comput., vol. 36, pp. 630-637, 1998.

[15] A. Marty and E. Neher, "Tight seal whole cell recording," in Single Channel Recording, B. Sakmann and E. Neher, Eds. New York: Plenum, 1983, pp. 107-122.

[16] S. A. Fedulova, D. V. Vasilyev, and N. S. Veselovsky, "Voltage-operated potassium currents in the somatic membrane of rat dorsal root ganglion neurons: Ontogenetic aspects," Neurosc., vol. 85, pp. 497-508, 1998.

[17] M. D. Baker and H. Bostock, "Low-threshold, persistent sodium curren in rat large dorsal root ganglion neurons in culture," J. Neurophysiol., vol. 77, pp. 1503-1513, 1997.

[18] B. Everill, M. A. Rizzo, and J. D. Kocsis, "Morphologically identified cutaneous afferent DRG neurons express three different potassium currents in varying proportions," J. Neurophysiol., vol. 79, pp. 1814-1824, 1998.

[19] Y. S. Kim, Y. K. Shin, C. S. Lee, and J.-H. Song, "Block of sodium currents in rat dorsal roots ganglion neurons by diphenhydramine," Brain Res., vol. 881, pp. 190-198, 2000.

[20] M. Bove, M. Grattarola, S. Martinoia, and G. Verreschi, "Interfacing cultured neurons to planar substrate microelectrodes: Characterization of the neuron-to-microelectrode junction," Bioelectrochem. Bioenerg., vol. 38, pp. 255-265, 1995.

[21] E. T. McAdams, A. Lackermeier, J. A. McLaughlin, D. Macken, and J. Jossinet, "The linear and nonlinear electrical properties of the electrode-electrolyte interface," Biosens. Bioelect., vol. 10, pp. 67-74, 1995.

[22] R. W. de Boer and A. van Oosterom, "Electrical properties of platinum electrodes: Impedance measurements and time-domain analysis," Med. Biol. Eng. Comput., vol. 16, pp. 1-10, 1978.

[23] W. G. Regehr, J. Pine, C. S. Cohan, M. D. Mischke, and D. W. Tank, "Sealing cultured invertebrate neurons to embedded dish electrodes facilitates long-term stimulation and recording," J. Neurosci. Meth., vol. 30, pp. 91-106, 1989.

[24] P. Fromherz, "Self-gating of ion channels in cell adhesion," Phys. Rev. Lett., vol. 78, pp. 4131-4134, 1997.

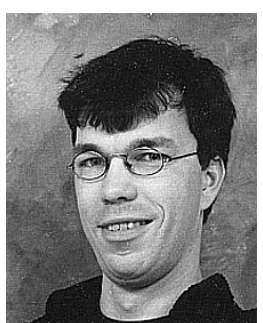

Jan Reinoud Buitenweg received the M.Sc. degree in biomedical engineering from the University of Twente, Enschede, The Netherlands, in 1998. He received the Ph.D. degree from the same institution in 2001 after studying the electrical behavior of the interface between cultured neurons and substrate embedded microelectrodes.

At present, he is an Assistant Professor with the University of Twente, with research interests in information exchange with the neuromuscular system.

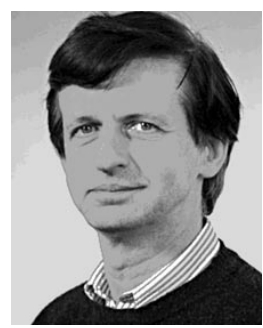

Wim L. C. Rutten received the Ph.D. degree in experimental physics from Leiden University, Leiden, The Netherlands, in 1979. Thereafter, he studied the auditory system at the ENT Department of Leiden University Hospital.

Since 1985, he has been with the BMTI/Faculty of Electrical Engineering of the University of Twente, Enschede, The Netherlands, as Assistant and Associate Professor of Biomedical Control Systems. His present research interests are neurotechnology (neuprocessing, and bioelectricity. roelectronic interfaces, and cultured probes), signal

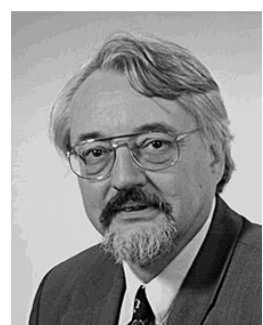

Enrico Marani received the Ph.D. degree in neuroanatomy from Leiden University, Leiden, The Netherlands, in 1982.

Thereafter, he became Head of the Neuroregulation Group, Department of Physiology, Leiden University. Since 1997, he is part-time Professor in neurophysiology at the BMTI/Faculty of Electrical Engineering of the University of Twente, Enschede, The Netherlands. His present research interests are neuroregulation and neurotechnology, especially in the field of nerve regeneration. 